International Journal of Pure and Applied Mathematics

Volume 88 No. 4 2013, 523-527

ISSN: 1311-8080 (printed version); ISSN: 1314-3395 (on-line version)

url: http://www.ijpam.eu

doi: http://dx.doi.org/10.12732/ijpam.v88i4.6

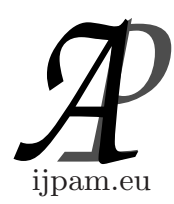

\title{
WEAK-STAR CONVERGENCE OF MEASURES
}

\author{
Kifah Y. Al-Hami \\ Department of Mathematics \\ University of Bahrain \\ P.O. Box 32038, Sukhair, BAHRAIN
}

Abstract: Consider a lebesgue measure $\lambda$ on $[0,1]$ and let $0 \leq h \in L^{1}(\lambda)$. We construct a measure $\mu$ such that $\|\mu\| \leq 2$. $\|h\|_{L^{1}(\lambda)}, \mu \perp \lambda$ and $\mu(E)=0$, for every Carleson set $E$ in $[0,1]$ and converges weak-star to $h d \lambda$.

AMS Subject Classification: Carleson set, weak-star convergence, Cantor set, $L^{1}(\lambda)$

\section{Introduction}

For the convenience of the reader, we introduce the following definition and example.

Definition 1.1. A subset $E$ of $\partial D$ is called a Carleson set if $E$ is closed with Lebesgue measure zero and $\sum_{v}\left|I_{v}\right| \log \frac{1}{\left|I_{v}\right|}<\infty$, where $\left\{I_{v}\right\}$ are the complementary arcs of $E$

Example. Let $E$ be the Cantor set on $[0,1]$. It can be easily seen that:

1. $E$ is closed,

2. $E$ is of Lebesgue measure zero, and

3. $[0,1] \backslash E$ is the pairwise disjoint union $\bigcup_{k=0}^{\infty}\left(\bigcup_{n=1}^{2^{k}} I_{n, k}\right)$, where each $I_{n, k}$

Received: July 31, 2013

(c) 2013 Academic Publications, Ltd. url: www.acadpubl.eu 
has length $\frac{1}{3^{k+1}}$. Therefore:

$$
\begin{aligned}
\sum m\left(I_{k}\right) \log \left(\frac{1}{m\left(I_{k}\right)}\right) & =\sum_{k=0}^{\infty} \frac{2^{k}}{3^{k+1}} \cdot \log \left(\frac{1}{\left(\frac{1}{3^{k+1}}\right)}\right) \\
& =\frac{1}{3} \sum_{k=1}^{\infty}(k+1) \cdot\left(\frac{2}{3}\right)^{k} \log (3)<\infty
\end{aligned}
$$

by the Ratio Test. Consequently, $E$ is a Carleson subset of $[0,1]$.

\section{Main Result}

Let $\lambda$ denote Lebesgue measure on $[0,1]$ and suppose that $0 \leq h \in L^{1}(\lambda)$. Then, for any $\varepsilon>0$ and $C_{\circ} \geq 1$ there exists a measure $\mu$ such that $\|\mu\| \leq$ 2. $\|h\|_{L^{1}(\lambda)}, \mu \perp \lambda, \mu(E)=0$ for every Carelson set $E$ in $[0,1]$, and

$$
\left|\int_{[0,1]}(g d \mu-g h d \lambda)\right| \leq \varepsilon,
$$

whenever $0 \leq g \in C^{\infty}([0,1])$ and $\|g\|_{\infty},\|g\|_{\infty} \leq C_{\circ}$.

Proof. Choose $\varepsilon, 0<\varepsilon<1$. Since $\|g\|_{\infty} \leq C_{\circ}$, we can apply the MeanValue Theorem and find a partition $\left\{x_{i}: i=0, \ldots, M\right\}$ of $[0,1]$ that depends only on the constant $C_{\circ}$ and a step function $g^{*}=\sum_{i=1}^{M} \alpha_{i} \chi_{F_{i}} \quad\left(F_{i}:=\left[x_{i-1}, x_{i}\right]\right)$ such that

$$
\left\|g-g^{*}\right\|_{\infty} \leq \frac{\varepsilon}{4}
$$

and $\left|\alpha_{i}\right| \leq C_{\circ}$ for $i=1, \ldots, M$. Since $h$ is integrable ( we may assume that $\left.\|h\|_{L^{1}(\lambda)}=1\right)$ there exists a step function $h^{*}=\sum_{j=1}^{N} \beta_{j} \chi_{B_{j}}$, where the $B_{j^{\prime} s}$ are intervals that form a partition of $[0,1]$ such that

$$
\left|\int_{[0,1]}\left(h^{*} d \lambda-h d \lambda\right)\right| \leq \frac{\varepsilon}{4 M C_{\circ}} .
$$


Also, there exists $\delta>0$ such that whenever $A \subseteq[0,1]$ with $\lambda(A) \leq \delta$, then

$$
\int_{A} h^{*} d \lambda \leq \frac{\varepsilon}{16 M C_{\circ}} .
$$

Start with a measure $\eta$ on $[0,1]$ such that $\eta \perp \lambda, \eta([0,1])=1$ and $\eta(E)=0$ for every Carleson set $E$ in [0,1] ( see [1] ). Let $K$ be a large enough fixed integer such that $\frac{1}{K} \leq \delta$ and let $A_{k}=\left[\frac{k-1}{K}, \frac{k}{K}\right], k=1, \ldots, K$. It is clear that $\bigcup_{k=1}^{K} A_{k}=[0,1]$ and $\lambda\left(A_{k}\right)=\frac{1}{K}$. For $0<t<1$, define $\eta_{t}$ on the Borel subsets $B$ of $[0,1]$ by $\eta_{t}(B)=\eta\left(\frac{1}{t} B\right)$. Define $\mu_{k}$ on the Borel subsets $B$ of $A_{k}$ by $\mu_{k}(B)=\eta_{1 / K}\left(B-\left(\frac{k-1}{K}\right)\right) \quad(k=1, \ldots, K)$. Notice that $\mu_{k}\left(A_{j}\right)=0$, whenever $k \neq j$ and $\mu_{k}\left(A_{k}\right)=1$, for $k=1,2, \ldots, K$. Now, if $E$ is a Carleson set, then so is $\alpha E-\beta$ for any constants $\alpha, \beta$, and hence $\mu_{k}(E)=0$. Let $\mu=\sum_{k=1}^{K} c_{k} \mu_{k}$ where $c_{k}=\sum_{j=1}^{N} \beta_{j} \lambda\left(B_{j} \cap A_{k}\right)$. Then $\mu(E)=0$ for every Carleson set $E$ in $[0,1]$ and

$$
\begin{aligned}
\mu([0,1]) & =\sum_{k=1}^{K} c_{k} \mu_{k}([0,1])=\sum_{k=1}^{K} \sum_{j=1}^{N} \beta_{j} \lambda\left(B_{j} \cap A_{k}\right) \\
& =\sum_{j=1}^{N} \beta_{j}\left(\sum_{k=1}^{K} \lambda\left(B_{j} \cap A_{k}\right)\right)=\sum_{k=1}^{K} \beta_{j} \lambda\left(B_{j}\right)=\int_{[0,1]} h^{*} d \lambda \\
& \leq 2\|h\| L^{1}(\lambda) .
\end{aligned}
$$

Let $F$ be any closed subinterval of $[0,1]$, and let

$$
\Delta=\left\{k: k=1, \ldots, K \text { and } A_{k} \subseteq F\right\} .
$$

Now, there are at most two intervals $A_{k_{\circ}}, A_{k_{1}}$ such that $F \cap A_{k_{i}} \neq \emptyset$ for $i=0,1$ and yet $k_{0}, k_{1} \notin \Delta$. Then

$$
\begin{aligned}
\int_{F}\left(d \mu-h^{*} d \lambda\right) & =\mu(F)-\sum_{j=1}^{N} \beta_{j} \lambda\left(B_{j} \cap F\right) \\
& =\sum_{k=1}^{K} c_{k} \mu_{k}\left(A_{k} \cap F\right)-\sum_{j=1}^{N} \beta_{j} \lambda\left(B_{j} \cap F\right)
\end{aligned}
$$




$$
\begin{aligned}
= & \sum_{k \in \Delta} \sum_{j=1}^{N} \beta_{j} \lambda\left(B_{j} \cap A_{k}\right) \cdot \mu_{k}\left(A_{k} \cap F\right)-\sum_{j=1}^{N} \beta_{j} \lambda\left(B_{j} \cap\left(\bigcup_{k \in \Delta} A_{k}\right)\right) \\
& +\sum_{j=1}^{N} \beta_{j} \lambda\left(B_{j} \cap A_{k_{\circ}}\right) \cdot \mu_{k_{\circ}}\left(A_{k_{\circ}} \cap F\right) \\
& +\sum_{j=1}^{N} \beta_{j} \lambda\left(B_{j} \cap A_{k_{1}}\right) \cdot \mu_{k_{1}}\left(A_{k_{1}} \cap F\right) \\
& -\sum_{j=1}^{N} \beta_{j} \lambda\left(B_{j} \cap F \cap A_{k_{\circ}}\right)-\sum_{j=1}^{N} \beta_{j} \lambda\left(B_{j} \cap F \cap A_{k_{1}}\right) \\
= & \sum_{j=1}^{N} \beta j \lambda\left(B_{j} \cap A_{k_{\circ}}\right) \cdot \mu_{k_{\circ}}\left(A_{k_{\circ}} \cap F\right) \\
& +\sum_{j=1}^{N} \beta_{j} \lambda\left(B_{j} \cap A_{k_{1}}\right) \cdot \mu_{k_{1}}\left(A_{k_{1}} \cap F\right) \\
& -\sum_{j=1}^{N} \beta_{j} \lambda\left(B_{j} \cap F \cap A_{k_{\circ}}\right)-\sum_{j=1}^{N} \beta_{j} \lambda\left(B_{j} \cap F \cap A_{k_{1}}\right) .
\end{aligned}
$$

Therefore

$$
\left|\int_{F}\left(d \mu-h^{*} d \lambda\right)\right| \leq 2\left[\int_{A_{k_{\circ}}} h^{*} d \lambda+\int_{A_{k_{1}}} h^{*} d \lambda\right] \stackrel{b y(3)}{\leq} \frac{\varepsilon}{4 M C_{\circ}} .
$$

Now

$$
\begin{aligned}
\left|\int_{[0,1]}(g d \mu-g h d \lambda)\right| \leq & \int_{[[0,1]}\left|g-g^{*}\right| d \mu+\left|\int_{[0,1]} g^{*}(d \mu-h d \lambda)\right| \\
& +\int_{[0,1]}\left|g-g^{*}\right| h d \lambda \\
\qquad y(1) \frac{\varepsilon}{2}+\sum_{i=1}^{M}\left|\alpha_{i}\right|\left|\int_{F_{i}} d \mu-h d \lambda\right| \leq \frac{\varepsilon}{2} & \\
& +M C_{\circ}\left|\int_{F_{i}} d \mu-h d \lambda\right|
\end{aligned}
$$




$$
\underset{b y(2) \&(4)}{\leq} \leq \frac{\varepsilon}{2}+M C_{\circ}\left[\left|\int_{F_{i}} d \mu-h^{*} d \lambda\right|+\left|\int_{F_{i}}\left(h^{*} d \lambda-h d \lambda\right)\right|\right\rfloor
$$

\section{References}

[1] K. Al-hami, Singular inner cyclic vector in Bergman space, Journal Applied Math. Inf. Sci., 1, No. 4 (2010), 1FT-5FT, 
